\title{
Evaluación de la Iniciativa Personal en el Ámbito Educativo y su Relación con la Actitud Emprendedora y el Rendimiento Académico
}

\author{
Personal Initiative Assessment in the Educational Field and its Relationship with \\ Entrepreneurial Attitude and Academic Achievement
}

\author{
Arantxa Gorostiaga ${ }^{1}$, Nekane Balluerka ${ }^{2}$, Imanol Ulacia ${ }^{3}$ y Jone Aliri ${ }^{4}$
}

\begin{abstract}
Resumen
A pesar de la relevancia de la iniciativa personal en la educación, no existen instrumentos en lengua vasca para la evaluación de dicha variable. Por ello, el objetivo del estudio consistió en elaborar y validar un instrumento para evaluar la iniciativa personal en el ámbito educativo. La muestra estuvo compuesta por 278 estudiantes de Formación Profesional. El cuestionario reveló una estructura factorial que incluía tres componentes (Proactividad-Prosocialidad, Persistencia y Autoinicio) con índices aceptables de consistencia interna y buena validez convergente en relación a la Self-Reported Initiative Scale. Asimismo, se obtuvieron evidencias de validez de relación con otras variables. Los resultados indicaron que este instrumento permite evaluar de forma adecuada la iniciativa personal en estudiantes y que dicha variable se relaciona con la actitud emprendedora y el rendimiento académico. Se plantea su utilidad para la evaluación de programas de intervención que traten de promover la iniciativa personal en el ámbito educativo.
\end{abstract}

Palabras clave: iniciativa personal, evaluación educativa, elaboración de test, actitud emprendedora, rendimiento académico

\begin{abstract}
Despite the importance of the personal initiative in education, there are no measures to assess this variable in Basque language. Consequently, the objective of the study was to develop and validate a questionnaire to assess personal initiative in the educational field. The sample comprised 278 vocational training students. The results showed that the questionnaire had a three factor structure (proactivity-prosocial behaviour, persistence and self-starting) with acceptable internal consistency indices and good convergent validity in relation to the Self-Reported Initiative Scale. Additionally, evidence of validity based on relationship to other variables was also obtained. The results indicate that this new measure is very useful for assessing personal initiative among students and that this variable is related with entrepreneurial attitude and academic achievement. The usefulness of the developed instrument for the evaluation of intervention programs seeking to promote personal initiative in education is concluded.
\end{abstract}

Keywords: personal initiative, educational assessment, test construction, entrepreneurial attitude, academic achievement

Agradecimientos: El presente estudio fue parcialmente financiado por el Programa para la promoción de una sociedad emprendedora (OF83/2010) de la Diputación Foral de Gipuzkoa, así como por el Vicerrectorado de Investigación de la Universidad del País Vasco/Euskal Herriko Unibertsitatea (Subvención General a Grupos de Investigación, GIU14/18).

\footnotetext{
${ }^{1}$ Doctora en Psicología por la Universidad del País Vasco. Profesora Titular de Universidad, Universidad del País Vasco UPV/EHU. Facultad de Psicología. Departamento de Psicología Social y Metodología de las Ciencias del Comportamiento Avda. Tolosa, 70. 20018 - Donostia-San Sebastián. Correo: arantxa.gorostiaga@ehu.eus

${ }^{2}$ Doctora en Psicología por la Universidad del País Vasco. Profesora Catedrática, Universidad del País Vasco UPV/EHU. Correo: nekane.balluerka@ehu.eus

${ }^{3}$ Doctor en Psicología por la Universidad del País Vasco. Profesor Adjunto, Universidad del País Vasco UPV/EHU. Correo: imanol.ulacia@ehu.eus

${ }^{4}$ Doctora en Psicología por la Universidad del País Vasco. Profesora Adjunta. Universidad del País Vasco UPV/EHU. Correo: jone.aliri@ehu.eus
} 


\section{Introducción}

Partiendo de conceptos clásicos de la teoría de la motivación, Frese y Fay (2001) definieron la iniciativa personal como un síndrome conductual que caracteriza a las personas autoiniciadoras, proactivas y persistentes a la hora de superar las dificultades que emergen en la consecución de los objetivos. Entendemos síndrome como un conjunto de fenómenos que caracterizan una situación determinada, es decir, como un conjunto de conductas y no una única conducta aislada. El autoinicio implica que es uno/a mismo/a quien decide hacer algo, sin recibir ningún tipo de orden o de instrucción explícita. Uno/a mismo/a establece sus propios objetivos y se responsabiliza de ellos. La proactividad significa que la persona detecta los problemas y las oportunidades de forma anticipada, desarrollando estrategias para afrontar tales problemas y aprovechar las oportunidades. Implica visión a largo plazo y búsqueda activa de feedback. La persistencia se refiere a la obstinación en la consecución de los objetivos auto-iniciados, a la continuación de la acción a pesar de las dificultades y a la firme voluntad de seguir avanzando. Es importante subrayar que las conductas que carecen de una orientación prosocial no pueden constituir iniciativa personal. Para ser considerada iniciativa personal, una conducta autoiniciada debe beneficiar no solo al propio sujeto, sino también a su grupo de pertenencia o entorno más próximo (Frese \& Fay, 2001). No en vano, la conducta prosocial puede definirse como una conducta de carácter voluntario que resulta beneficiosa para los demás (Martorell, González, Ordóñez, \& Gómez, 2011).

Numerosos estudios sugieren que el desarrollo de la iniciativa personal influye positivamente tanto en los individuos como en las organizaciones. Por una parte, las personas con elevados niveles de iniciativa personal obtienen mejores resultados académicos (Fay \& Frese, 2001; Frese \& Fay, 2001), desempeñan mejor sus tareas (Frese, 2001; Frese \& Zapf, 1994; Lisbona \& Frese, 2012), son más empleables (Fay \& Frese, 2001; Frese, Fay, Hilburger, Leng, \& Tag, 1997; Lantz \& Anderson, 2009), más innovadoras (Baer \& Frese, 2003; Lisbona, Palací, \& Gómez, 2008; Rank, Boedeker, Linke, \& Frese, 2004;
Rank, Pace, \& Frese, 2004) y más emprendedoras (Frese, 2009; Frese et al., 1997; Kraus, Frese, Friedrich, \& Unger, 2005; Lisbona \& Frese, 2012). Por otra parte, las organizaciones que promueven la iniciativa personal responden con mayor éxito a nuevos retos (Frese \& Fay, 2001; Frese, Van Gelderen, \& Ombach, 2000; Koop, De Reu, \& Frese, 2000; Lumpkin \& Dess, 1996), son más eficientes (Fay \& Frese, 2001; Frese, 2000; Frese \& Fay, 2001; Motowidlo \& Scotter, 1994) y más rentables (Baer \& Frese, 2003).

Aunque fue originado en el ámbito organizacional (Frese et al., 1997), el concepto de iniciativa personal puede ser aplicado en diversos contextos, entre los que cabría destacar el de la educación (Fay \& Frese, 2000; Frese \& Fay, 2001). Según Lisbona y Frese (2012), existen tres razones principales para aplicar el enfoque de la iniciativa personal al contexto académico. En primer lugar, la concepción activa que subyace a la iniciativa personal es la que mejor se adecua a la naturaleza activa del ser humano, y la que puede fomentar en mayor medida las potencialidades de los/as estudiantes (Frese \& Fay, 2001; González \& Tourón, 1992; Lisbona \& Frese, 2012). En segundo lugar, existen evidencias de que la iniciativa personal contribuye al éxito académico (Fay \& Frese, 2001; Frese \& Fay, 2001). En tercer lugar, si el actual mercado laboral necesita trabajadores/as activos/as, responsables e iniciadores/as, la mejor manera de promover este tipo de trabajadores/as consiste en comenzar a trabajar esta competencia en la escuela. Cabe suponer que el desarrollo de la iniciativa personal de los/as estudiantes, les facilitará el acceso al empleo y les permitirá adaptarse de forma más adecuada a las exigencias del mercado laboral. Por otra parte, la iniciativa personal constituye una de las competencias clave del Marco de referencia europeo para el aprendizaje permanente $\mathrm{y}$, en consecuencia, una de las competencias básicas de diversos sistemas educativos. Las competencias clave son aquellas que todas las personas precisan para su realización y desarrollo personales, así como para la ciudadanía activa, la inclusión social y el empleo (Comisión Europea, 2007). El hecho de que los principales órganos gestores de diversos sistemas educativos internacionales planteen políticas y actuaciones específicas para el desarrollo de la 
iniciativa personal, constituye una de las principales evidencias de la relevancia de dicho constructo a nivel educativo.

En diversos estudios se ha observado que la iniciativa personal se relaciona con una serie de variables que son relevantes en el ámbito educativo, tales como la actitud emprendedora, el rendimiento académico, la autoeficacia, las aspiraciones de control y responsabilidad y la dimensión de responsabilidad de la personalidad. En lo que respecta a la actitud emprendedora, algunos autores han encontrado que los emprendedores presentan puntuaciones más altas que los no emprendedores en autoinicio y persistencia (Frese et al., 1997) y que la iniciativa personal correlaciona con el éxito emprendedor (Korunka, Frank, Lueger, \& Mugler, 2003; Krauss et al., 2005). En relación al rendimiento académico, diversos estudios han constatado una relación positiva entre la iniciativa personal y las calificaciones en la licenciatura y en educación secundaria (Fay \& Frese, 2001; Frese \& Fay, 2001). Además, la iniciativa es el único factor resiliente que se asocia al rendimiento académico en estudiantes universitarios/as y el que más lo determina (Peralta, Ramírez, \& Castaño, 2006). Los trabajos sobre aprendizaje autorregulado (Zimmerman \& Labuhn, 2012), pasión armoniosa hacia los estudios (Lisbona \& Frese, 2012) y autoestima y autoconcepto (González \& Tourón, 1992), aportan evidencias complementarias en este sentido. En cuanto a la autoeficacia, se ha encontrado que esta variable se relaciona con la probabilidad de llevar a cabo conductas de iniciativa personal (Frese, Teng, \& Wijnen, 1999; Sanjuán, Pérez, \& Bermúdez, 2000) y ejerce una gran influencia en la elección de actividades y en la perseverancia de las personas cuando se enfrentan a determinados retos (Chávez, Peinado, Ornelas, \& Blanco, 2013). En lo que se refiere a las aspiraciones de control y responsabilidad, algunos autores aportan evidencias que vinculan tales aspiraciones a la iniciativa personal (Frese \& Fay, 2001; Lisbona, Palací, \& Agulló, 2008). Finalmente, en un estudio reciente se encontró una relación positiva entre la iniciativa personal y la responsabilidad, como rasgo de personalidad (Autores, 2014).

Dada la relevancia de la iniciativa personal en el ámbito académico y la escasez de medidas para evaluar la iniciativa personal de los estudiantes (Balluerka et al., 2014), el objetivo de este trabajo consistió en diseñar y validar un instrumento en lengua vasca para evaluar la iniciativa personal en la formación profesional inicial. Como se ha señalado previamente, hay una serie de variables, también relevantes en el ámbito educativo, que han mostrado tener relación con la iniciativa personal, por lo que dichas variables van a ser utilizadas como referencia en la validación del instrumento.

\section{Elaboración del instrumento y estudio piloto}

Atendiendo al modelo teórico de cuatro dimensiones de la iniciativa personal (Frese \& Fay, 2001), se formularon 35 ítems iniciales. Dichos ítems fueron elaborados a partir de adaptaciones y reformulaciones de los ítems de diversos cuestionarios que, debido a sus características no constituyen instrumentos idóneos para evaluar la iniciativa personal en el ámbito educativo en el País Vasco (Autores, 2014). Dichos instrumentos son los siguientes: Interview and questionnaire based scales for measuring personal initiative (Frese et al., 1996); Situational Judgement Test of Personal Initiative (Bledow, \& Frese, 2009); Self-Reported Initiative (Frese et al., 1997); Inventory of Learner Initiative (Ponton \& Carr, 2002); Scale of Work Climate focused on initiative (Baer \& Frese, 2003). Así, se elaboraron 16 ítems a partir de la dimensión de autoinicio, 6 ítems a partir de la dimensión de proactividad, 7 ítems a partir de la dimensión de persistencia y 6 ítems a partir de la dimensión de conducta prosocial. Del total de ítems, 20 se redactaron en sentido positivo, es decir, un mayor acuerdo con el ítem indicaría mayor nivel de iniciativa personal $\mathrm{y}$, el resto, en sentido inverso. Una vez construida la batería inicial de ítems se procedió a su evaluación por parte de un grupo de jueces expertos/as con el objetivo de proporcionar evidencias de que dichos ítems eran relevantes para la iniciativa personal y representaban adecuadamente a cada una de sus dimensiones. El grupo de expertos/as estuvo compuesto por 8 profesores/as, 2 directores/as de centro y 2 expertos/as en innovación y emprendizaje. Aunque el porcentaje de asignación correcta de algunos de los ítems fue bajo, las valoraciones medias en adecuación fueron elevadas para 
prácticamente todos los ítems, por lo que en esta fase se decidió no modificar ni eliminar ningún ítem.

Tras la consulta a expertos/as, se procedió a llevar a cabo un estudio piloto. En la primera fase, el cuestionario fue administrado a 44 estudiantes de Ingeniería en Alternancia (41 hombres y 3 mujeres), con un rango de edad comprendido entre los 21 y los 39 años $(M=25.1 ; D T=4.3)$. En esta fase se realizaron una serie de análisis cuantitativos (cálculos de índices de correlación ítem-total y estadísticos descriptivos) y cualitativos (análisis de la dificultad para comprender algunos ítems) que dieron lugar a la eliminación de 10 ítems y a la reformulación de otros 10. En la segunda fase, el cuestionario fue administrado a 176 estudiantes de formación profesional inicial (123 hombres y 53 mujeres), con un rango de edad que osciló entre los 15 y los 30 años $(M=19.88 ; D T=3.04)$. El objetivo en este caso consistió en determinar el número de dimensiones del cuestionario y los ítems que componen tales dimensiones. Para ello se realizó un análisis de componentes principales con rotación oblicua sobre los 25 ítems del cuestionario. El procedimiento MAP (Minimum Average Partial; Velicer, 1976) utilizado para establecer el número de dimensiones del cuestionario, sugirió la existencia de tres dimensiones que explicaban, en total, el $36.88 \%$ de la varianza. Posteriormente, se seleccionaron aquellos ítems que presentaron un peso factorial igual o superior a $.45 \mathrm{y}$ que saturaban claramente en una única dimensión. De esta manera, el instrumento quedó compuesto por 17 ítems. El factor 1 (valor propio $=3.82$ ) constaba de 8 ítems y explicaba el $22.48 \%$ de la varianza. Tales ítems pertenecían a los componentes de proactividad y conducta prosocial. El factor 2 (valor propio = 2.35) explicaba el 13.84 de la varianza y sus 4 ítems conformaban la dimensión de persistencia. El factor 3 (valor propio $=1.74$ ) estaba compuesto por 5 ítems pertenecientes al componente de autoinicio y explicaba el $10.24 \%$ de la varianza. El instrumento recibió la siguiente denominación: Escala para la medición de la iniciativa personal en el ámbito educativo-Euskera (EMIPAE-E).

\section{Método}

\section{Muestra}

La muestra estuvo compuesta por 278 participantes (194 hombres y 84 mujeres) con una edad comprendida entre los 15 y los 30 años $(M=19.88 ; D T=2.93)$. Tales participantes cursaban sus estudios de ciclos de grado medio o superior de formación profesional en 9 centros educativos ubicados en la Comunidad Autónoma del País Vasco. El estudio se centró en la población de estudiantes de Formación Profesional por su clara vinculación con el ámbito laboral, en el que la iniciativa personal constituye una competencia muy relevante. El $44.3 \%$ de los/as estudiantes tenía experiencia laboral. En cuanto a su nivel socioeconómico, la mayoría de la muestra (59.5\%) pertenecía a un nivel medio, el $30.8 \%$ a un nivel medio-bajo, el $8.1 \%$ a un nivel alto y, finalmente, el $1.6 \%$ a un nivel socioeconómico bajo. La selección de la muestra fue incidental. Con respecto a su tamaño, se estimó que para llevar a cabo un AFC con tres dimensiones, con cargas factoriales de .65 y un mínimo de tres o cuatro indicadores por factor, sería necesaria una muestra de, al menos, 230 participantes (Wolf, Harrington, Clark, \& Miller, 2013).

\section{Instrumentos}

Los instrumentos utilizados fueron los siguientes:

Escala para la medición de la iniciativa personal en el ámbito educativo (EMIPAE-E). La versión final del EMIPAE-E consta de 17 ítems que evalúan tres dimensiones de la iniciativa personal: a) la proactividad y la conducta prosocial (p.e. Ikasgelan / Tailerrean / Laborategian gogotsu parte hartzen dut, trukean inolako ordainsaririk jasotzen ez badut ere [Suelo participar activamente en el Aula / Taller / Laboratorio, aunque no obtenga una recompensa a cambio]), b) la persistencia (p.e. Modulu/Proiektu/Ikasgai baten edukinak ulertzen ez ditudanean, desesperatu egiten naiz eta etsi egiten dut [Cuando empiezo a no entender los contenidos de algún Módulo / Proyecto / Asignatura, me desespero y abandono]-ítem inverso) y c) el autoinicio (p.e. Ikasgelan / Tailerrean / Laborategian bururatzen zaizkidan ideiak praktikara eramaten bereziki ona naiz [Soy 
especialmente bueno/a llevando a la práctica las ideas que se me ocurren en el Aula / Taller / Laboratorio]). Los ítems se responden en una escala Likert de 5 puntos que oscilan entre 1 (Totalmente en desacuerdo) y 5 (Totalmente de acuerdo). El EMIPAE-E se puede solicitar a los/as autores/as.

Self-Reported Initiative (Frese et al., 1997). Se trata de una medida de autoinforme para la evaluación de la iniciativa personal. Consta de 7 ítems (p.e. Afronto los problemas de forma activa) que se responden en una escala Likert de 5 puntos que oscilan entre 1 (Totalmente en desacuerdo) y 5 (Totalmente de acuerdo). Tales ítems miden principalmente el componente de autoinicio de la Iniciativa Personal, aunque también hay ítems que hacen referencia a la persistencia, y fueron traducidos al español para un estudio anterior. En dicho estudio, el instrumento presentó una estructura unidimensional y un índice de consistencia interna de .71 (Balluerka et al., 2014). En la muestra actual, el índice de consistencia interna fue de .83 .

Escala de Actitud Emprendedora (Roth \& Lacoa, 2009). Se trata de una escala unidimensional destinada a evaluar la actitud emprendedora a partir de afirmaciones relacionadas con la proactividad, la disposición a la excelencia, la búsqueda de la eficiencia, la confianza en el éxito y la capacidad resiliente. Consta de 15 ítems (p.e. Estoy siempre dispuesto a emprender nuevos proyectos) que se responden en una escala Likert de 4 puntos que oscilan entre 1 (Totalmente en desacuerdo) y 4 (Totalmente de acuerdo). El instrumento presenta una estructura unidimensional y adecuada validez de relación con otras variables (Roth \& Lacoa, 2009). Dado que la escala fue creada para su aplicación en población boliviana, en un estudio anterior (Autores, 2014) se realizaron pequeñas modificaciones en 3 de los ítems para adecuarlos a nuestro contexto cultural. $\mathrm{El}$ instrumento modificado presentó un índice de consistencia interna de .92. En la muestra actual, el índice de consistencia interna fue de .85 .

Escala de Autoeficacia General (Baessler \& Schwarzer, 1996; en su adaptación española, Sanjuán et al., 2000). Es un instrumento de autopercepción de la competencia personal para manejar de forma eficaz una gran variedad de situaciones estresantes. Consta de 10 ítems (p. e., Puedo resolver la mayoría de los problemas si me esfuerzo lo necesario) que se responden en una escala Likert de 10 puntos que oscilan entre 1 (Totalmente en desacuerdo) y 10 (Totalmente de acuerdo). La adaptación española presenta una estructura unidimensional, un índice de consistencia interna de $.87 \mathrm{y}$ una adecuada validez de relación con otras variables (Sanjuán et al., 2000). En la presente muestra, la consistencia interna fue de .89 .

Escala de Aspiraciones de Control y Responsabilidad (Frese, Erbe-Heinbokel, Grefe, Rybowiak, \& Weike, 1994; en su adaptación española, Lisbona, Palací, \& Agulló, 2008). Evalúa el grado en el que una persona puede decidir qué tareas lleva a cabo diariamente, establecer la cantidad de trabajo diario a realizar, establecer normas $y$ procedimientos acerca de cómo llevar a cabo las tareas y sentirse responsable de las tareas que realiza. Consta de 6 ítems inversos que se responden en una escala Likert de 5 puntos que oscilan entre 1 (Totalmente en desacuerdo) y 5 (Totalmente de acuerdo). Dado que la escala fue creada para su aplicación en el ámbito organizacional, en un estudio anterior se sustituyeron algunos términos específicos de dicho ámbito por otros más propios del ámbito educativo (p.e. En el ítem 6 se sustituyó el término supervisor/a por el de profesor/a, de forma que el ítem se formuló como "Prefiero tener un/a profesor/a que me diga exactamente lo que tengo que hacer, así él o ella serán los responsables si algo sale mal"). En dicho estudio el instrumento presentó una estructura unidimensional y un índice de consistencia interna de .78 (Balluerka et al., 2014). En la muestra actual, el índice de consistencia interna fue de .83 .

Adaptación vasca del Inventario NEO reducido de 5 factores (NEO-FFI; Balluerka, Gorostiaga, Alonso-Arbiol, \& Haranburu, 2008). Se trata de una versión de la Forma $S$ del NEO PI-R que ofrece una medida rápida y general de los 5 factores de la personalidad: "Neuroticismo", "Extraversión", "Apertura", "Amabilidad" y "Responsabilidad". Consta de 60 ítems que se responden en una escala de respuesta tipo Likert de 5 puntos $(\mathrm{A}=$ En total desacuerdo, $\mathrm{E}=$ Totalmente de acuerdo). En el presente 
estudio se ha utilizado únicamente la dimensión de Responsabilidad (e.g. Nahiko ongi antolatzen dut nere burua zereginak garaiz amaitzeko $[\mathrm{Me}$ organizo bastante bien para finalizar mis tareas a tiempo]). El instrumento posee adecuada validez factorial, validez convergente, estabilidad temporal y consistencia interna. Concretamente, la consistencia interna de la dimensión de Responsabilidad fue de .86 (Balluerka et al., 2008). En la presente muestra, dicho índice fue de .71 .

Cuestionario sobre el rendimiento académico. Con el objeto de recabar las calificaciones académicas de los/as estudiantes se elaboró un breve cuestionario con los siguientes apartados: fecha de nacimiento de la alumno/a; listado de asignaturas a evaluar; y calificaciones finales obtenidas.

\section{Procedimiento}

El EMIPAE-E, junto con los instrumentos necesarios para su validación, fue aplicado por un psicólogo en el propio aula de los/as estudiantes y dentro del horario lectivo. Los instrumentos fueron administrados en diferentes sesiones y en el siguiente orden: EMIPAE-E, Escala de Actitud Emprendedora, Escala de Autoeficacia General, Self-Reported Initiative, Escala de Aspiraciones de Control y Responsabilidad y Adaptación vasca del Inventario NEO reducido de 5 factores. Al finalizar el curso escolar, los/as jefes/as de estudios de los centros educativos implicados cumplimentaron el cuestionario sobre el rendimiento académico. El estudio contó con el informe favorable de la Comisión de Ética para la Investigación y la Docencia (CEID) de la Universidad del País Vasco. Se solicitó consentimiento informado a los/as directores/as de los centros educativos, a los/as padres/madres y/o tutores/as de los/as participantes menores de edad y a los/as participantes mayores de edad.

\section{Análisis de datos}

La dimensionalidad del instrumento se examinó mediante un Análisis Factorial Confirmatorio (AFC) para tratar de confirmar la estructura de tres factores encontrada en el análisis factorial exploratorio llevado a cabo con la primera muestra. El método de estimación fue el Weighted Least Squares Mean and Variance adjusted (WLSMV). Dado que el ajuste de este modelo no resultó adecuado, se llevó a cabo un Modelo Exploratorio de Ecuaciones Estructurales (ESEM, Exploratory Structural Equation Modeling) tridimensional con rotación Target. El modelo ESEM (Marsh, Liem, Martin, Morin, \& Nagengast, 2011; Marsh, Morin, Parker, \& Kaur, 2014) constituye una buena alternativa de análisis cuando no se obtienen adecuados índices de bondad de ajuste en el AFC. A diferencia del AFC, en el ESEM, las saturaciones de los ítems en los factores que no les corresponden no se fijan a cero. Por lo tanto, se trata de una propuesta que constituye un híbrido entre el AFE y el AFC (Lloret-Segura, Ferreres-Traver, HernándezBaeza, \& Tomás-Marco, 2014). En el presente estudio, se utilizaron como índices de bondad de ajuste el Índice de ajuste comparativo (CFI), el Índice de Tucker-Lewis (TLI), el error cuadrático medio de aproximación (RMSEA) y la raíz cuadrática media residual ponderada (WRMR). En el caso del CFI y del TLI, valores superiores a .90 y .95 indican un ajuste aceptable y excelente, respectivamente. En cuanto al RMSEA, valores inferiores a .08 indican un ajuste aceptable, e inferiores a .06, un buen ajuste. Finalmente, en el caso de WRMR, valores inferiores a 1 reflejan un ajuste adecuado ( $\mathrm{Hu} \&$ Bentler, 1999). Posteriormente, se utilizó el coeficiente Alfa de Cronbach para estimar la consistencia interna de los factores derivados de dicho análisis.

A su vez, con el objetivo de obtener evidencias de validez convergente del EMIPAEE, se examinó, utilizando el coeficiente de correlación Rho de Spearman, la asociación entre sus dimensiones y la iniciativa personal autopercibida evaluada mediante la Self-Reported Initiative. Por último, para la obtención de evidencias de validez de relación con otras variables del instrumento se analizó la asociación que presentaban las dimensiones del EMIPAE-E con la actitud emprendedora, el rendimiento académico, la autoeficacia general, las aspiraciones de control y responsabilidad y el factor de personalidad denominado responsabilidad utilizando, en todos los casos, el coeficiente de correlación Rho de Spearman. Se utilizó el software Mplus (v. 7.4) para el AFC y el ESEM y el software SPSS (v. 20) para el resto de análisis. 


\section{Resultados}

\section{Dimensionalidad y consistencia interna}

Los índices de bondad de ajuste de los modelos AFC y ESEM de tres factores se presentan en la Tabla 1. Como se puede observar, el modelo AFC no presentó un ajuste adecuado. Por su parte, los índices de bondad de ajuste para el modelo ESEM fueron satisfactorios. Las cargas factoriales estandarizadas resultantes del modelo ESEM se presentan en la Tabla 2. Todas las cargas resultaron estadísticamente significativas y superiores a .49 , excepto las correspondientes a dos ítems. Únicamente un ítem presentó una carga superior a .30 en dos factores.

En relación a la consistencia interna, los coeficientes Alfa fueron de .77, .76 y .60 para las dimensiones de proactividad-prosocialidad, persistencia y autoinicio, respectivamente.

\section{Validez convergente}

Los coeficientes de correlación entre las puntuaciones obtenidas por los/as participantes en las dimensiones del EMIPAE-E y en el SelfReported Initiative fueron de $.21, .21$ y .35 para proactividad-prosocialidad, persistencia y autoinicio, respectivamente.

\section{Validez de relación con otras variables}

En la Tabla 3 se presentan las correlaciones de las dimensiones del EMIPAE-E con la Actitud emprendedora, el Rendimiento académico, la Autoeficacia, las Aspiraciones de control y responsabilidad y el factor Responsabilidad de la personalidad.

Tabla 1. Índices de bondad de ajuste de los modelos AFC y ESEM del EMIPAE-E

\begin{tabular}{lcccccc}
\hline Modelos & $\chi^{2}$ & gl & CFI & TLI & RMSEA (IC 90\%) & WRMR \\
\hline AFC 3 dim. & 383.964 & 116 & .875 & .853 & $.091(.081-.101)$ & 1.372 \\
ESEM 3 dim. & 181.780 & 88 & .956 & .932 & $.062(.049-.075)$ & .729
\end{tabular}

Nota. $\chi^{2}$ : Chi cuadrado; gl: grados de libertad; CFI: índice de ajuste comparativo; TLI: índice de Tucker-Lewis; RMSEA: error cuadrático medio de aproximación; IC: intervalo de Confianza; WRMR: raíz cuadrática media residual ponderada.

Tabla 2. Cargas factoriales estandarizadas resultantes del modelo ESEM $(\mathrm{N}=276)$

\begin{tabular}{lccc}
\hline Ítems & $\mathbf{F 1}$ & $\mathbf{F 2}$ & $\mathbf{F 3}$ \\
\hline Ítem 2 & $\mathbf{. 5 1 7}$ & -.009 & .199 \\
Ítem 4 & $\mathbf{. 5 8 3}$ & .028 & .220 \\
Ítem 5 & $\mathbf{. 7 4 9}$ & .177 & -.338 \\
Ítem 8 & $\mathbf{. 4 9 2}$ & .038 & .228 \\
Item 10 & $\mathbf{. 8 1 7}$ & -.221 & -.009 \\
Ítem 11 & $\mathbf{. 5 7 8}$ & -.114 & .138 \\
Ítem 14 & $\mathbf{. 6 5 4}$ & -.005 & -.013 \\
Ítem 16 & $\mathbf{. 5 5 5}$ & .285 & -.166 \\
Ítem 7 & -.029 & $\mathbf{. 5 0 9}$ & .092 \\
Ítem 12 & .042 & $\mathbf{. 5 7 1}$ & .053 \\
Ítem 13 & .039 & $\mathbf{. 8 4 0}$ & .002 \\
Ítem 15 & .045 & $\mathbf{. 8 8 0}$ & .067 \\
Ítem 1 & -.210 & .253 & $\mathbf{. 6 3 3}$ \\
Ítem 3 & -.103 & .029 & $\mathbf{. 5 7 6}$ \\
Ítem 6 & .126 & -.093 & $\mathbf{. 5 4 3}$ \\
Ítem 9 & $\mathbf{. 3 4 8}$ & .190 & $\mathbf{. 3 3 6}$ \\
Ítem 17 & .221 & -.134 & $\mathbf{. 3 9 0}$
\end{tabular}

Nota. Se han destacado en negrita las cargas factoriales superiores a .300 . 
Tabla 3. Correlaciones de las dimensiones del EMIPAE-E con la Actitud emprendedora, el Rendimiento académico, la Autoeficacia, las Aspiraciones de control y responsabilidad y el factor de Responsabilidad de

la personalidad

\begin{tabular}{lccc}
\hline & $\begin{array}{c}\text { Proactividad/ } \\
\text { prosocialidad }\end{array}$ & Persistencia & Autoinicio \\
\hline Actitud emprendedora $(\mathrm{N}=127)$ & $.44^{* *}$ & $.44^{* *}$ & $.39^{* *}$ \\
Rendimiento académico $(\mathrm{N}=65)$ & $.29^{*}$ & $.38^{* *}$ & .10 \\
Autoeficacia $(\mathrm{N}=130)$ & .10 & $.37^{* *}$ & $.21^{*}$ \\
Aspiraciones de control y & $.27^{*}$ & $.38^{* *}$ & .21 \\
responsabilidad $(\mathrm{N}=76)$ & $.25^{* *}$ & -.04 & $.32^{* *}$ \\
Responsabilidad $(\mathrm{N}=146)$ & & &
\end{tabular}

Como cabe observar en la tabla, se obtuvieron correlaciones moderadas de la variable Actitud emprendedora con todas las dimensiones de la iniciativa personal; de las variables Rendimiento académico y Aspiraciones de control y responsabilidad con las dimensiones de proactividad/prosocialidad y persistencia; de la variable Autoeficacia con la dimensión de persistencia; y finalmente, del factor Responsabilidad de la personalidad con las dimensiones de proactividad/prosocialidad y autoinicio.

\section{Conclusiones}

El objetivo del presente estudio fue elaborar y validar un instrumento en lengua vasca para evaluar la iniciativa personal en la formación profesional inicial.

En relación a la dimensionalidad del instrumento, los resultados del ESEM confirmaron la estructura de tres factores obtenida en el análisis factorial exploratorio. Dos de los factores reflejan dos de los componentes del modelo de referencia, a saber, el autoinicio y la persistencia, y un tercero incluye ítems referidos a la proactividad y a la conducta prosocial. La agrupación de los ítems de estos dos componentes en un solo factor es coherente con la literatura científica existente, ya que diversas investigaciones en el ámbito organizacional consideran la conducta prosocial como un subtipo de proactividad (Belschak \& Den Hartog, 2010). Dicho subtipo se caracteriza por la búsqueda anticipada de problemas y oportunidades con el fin de beneficiar no solo al propio sujeto sino también a su entorno más próximo, en contraposición con la proactividad pro-self en cuyo caso la anticipación persigue únicamente el beneficio personal. De hecho, esta estructura coincide con la encontrada en la versión en castellano del instrumento (Balluerka et al., 2014). Todos los ítems del instrumento presentan cargas factoriales superiores a .49 en sus correspondientes factores, a excepción de dos de los ítems.

Los índices de consistencia interna de las dimensiones del EMIPA-E pusieron de manifiesto que el instrumento presenta una adecuada fiabilidad, aunque la consistencia interna del componente de autoinicio fue menor que la de los dos restantes. Este índice más bajo puede ser debido a que uno de los ítems pesa en dos factores.

El patrón de correlaciones observado entre las puntuaciones de las dimensiones del EMIPA-E y del Self-Reported Initiative proporcionó evidencias sobre la validez convergente del instrumento. La correlación con la dimensión de autoinicio fue la más elevada, dado que la mayoría de los ítems del Self-Reported Initiative evalúan dicho componente de la iniciativa personal.

Con respecto a la validez de relación con otras variables, los resultados observados en relación con la Actitud emprendedora son coherentes con la literatura, mostrando correlaciones positivas con las dimensiones de persistencia y autonicio (Frese et al., 1997). En nuestro estudio, además, se ha encontrado que dicha variable se relaciona con la tercera dimensión de la iniciativa personal, a saber, la conducta proactiva y prosocial.

A su vez, en consonancia con estudios anteriores, se ha observado una relación positiva de las variables Rendimiento académico (Fay \& Frese, 2001) y Aspiraciones de control y 
responsabilidad (Frese \& Fay, 2001; Lisbona, Palací, \& Gómez, 2008) con las dimensiones de iniciativa personal, aunque en el caso de la dimensión de Autoinicio las correlaciones presentan una magnitud reducida.

En el caso de la variable Autoeficacia, únicamente se encontraron correlaciones con la dimensión de persistencia, en línea con otros estudios que muestran una relación positiva entre ambas variables (Sanjuán et al., 2000). Este hecho puede ser debido a que la Autoeficacia está muy relacionada con la personalidad resistente y el afrontamiento centrado en la tarea, características que definen a las personas persistentes.

Finalmente, con respecto a la dimensión de Responsabilidad de la personalidad, los resultados de nuestro estudio han corroborado lo observado en un estudio anterior (Balluerka et al., 2014), en el que también se hallaron relaciones positivas de esta dimensión con las dimensiones de proactividad/prosocialidad y autoinicio. De hecho, la responsabilidad se asocia a la tendencia al sentido del deber, a la organización, el orden, la búsqueda de objetivos, la autodisciplina y la eficiencia y, en este sentido, el sentimiento de responsabilidad sobre las tareas y los objetivos es una constante en el modelo de antecedentes y consecuencias de la iniciativa personal (Frese \& Fay, 2001).

Con respecto a las limitaciones del estudio, la más importante se relaciona con la composición de la muestra que fue mayoritariamente masculina y algo reducida. Por ello, sería recomendable ampliar el estudio de validación incluyendo un mayor número de participantes $\mathrm{y}$, sobre todo, participantes del sexo femenino. No obstante, a pesar de esta limitación, los resultados obtenidos permiten concluir que el EMIPAE-E constituye un instrumento adecuado para evaluar la iniciativa personal en estudiantes de formación profesional inicial. Además, dadas las características de los ítems del cuestionario, el instrumento puede ser de utilidad en otros niveles educativos, además de en formación profesional. Consideramos que la información derivada de la aplicación de este instrumento puede resultar muy útil para diseñar, implementar y evaluar programas de intervención que traten de promover la iniciativa personal en el ámbito educativo.

\section{Referencias}

Baer, M., \& Frese, M. (2003). Innovation is not enough: Climate for initiative and psychological safety, process innovation, and firm performance. Journal of Organizational Behavior, 24, 45-68. doi: http://dx.doi.org/10.1002/job.179

Baessler, J., \& Schwarzer, R. (1996). Evaluación de la autoeficacia: Adaptación española de la escala de Autoeficacia General. Ansiedad y Estrés, 2, 1-8.

Balluerka, N., Gorostiaga, A., Alonso-Arbiol, I., \& Haranburu, M. (2008). Manual técnico de la adaptación al euskera del NEO PI-R (Forma S). Madrid: TEA Ediciones, S.A.

Balluerka, N., Gorostiaga, A., \& Ulacia, I. (2014). Assessing personal initiative among vocational training students: Development and validation of a new measure. Spanish Journal of Psychology, 17, 1-9. doi:http://dx.doi.org/10.107/sjp.2014.80

Belschak, F., \& Den Hartog, D. (2010). Pro-self, prosocial, and pro-organizational foci of proactive behaviour: Differential antecedents and consequences. Journal of Occupational and Organizational Psychology, 83, 475-498. doi: http://dx.doi.org/10.1348/096317909X439208

Bledow, R. \& Frese, M. (2009). A situational judgement test of personal initiative: Towards understanding construct based situational judgement tests. Personnel Psychology, 62, 229-258.

doi:http://dx.doi.org/10.1111/j.1744-6570.200 9.01137.x

Chávez, A., Peinado, J. E., Ornelas, M., \& Blanco, H. (2013). Composición factorial de una escala de autoeficacia en conductas académicas en universitarios de ingeniería. Revista Iberoamericana de Diagnóstico y Evaluación - e Avaliação Psicológica, 35, 7793.

Comisión Europea (2007). Competencias clave para el aprendizaje permanente. Un marco de referencia europeo. Luxemburgo: Oficina de Publicaciones Oficiales de las Comunidades Europeas.

Fay, D., \& Frese, M. (2000). Self-starting behavior at work: Toward a theory of personal initiative. En J. Heckhausen (Ed.), 
Motivational Psychology of Human Development (pp. 307-324). Amsterdam: Elsevier.

Fay, D., \& Frese, M. (2001). The concept of personal initiative: An overview of validity studies. Human Performance, 4(1), 97-124. doi: http://dx.doi.org/10.1207/S15327043HUP1401_06

Frese, M. (2000). Success and failure of microbusiness owners in Africa: A psychological approach. Westport, CT: Greenwood. doi: http://dx.doi.org/10.1026//0932-4089.45.3.169

Frese, M. (2001). Personal initiative (PI): The theoretical concept and empirical findings. En M. Erez, U. Kleinbeck, \& H. Thierry (Eds.), Work motivation in the context of a global economy (pp. 99-110). Mahwah: Erlbaum.

Frese, M. (2009). Towards a psychology of entrepreneurship. An action theory perspective. Foundation and Trends in Entrepreneurship, 5, 437-494. doi: http://dx.doi.org/10.1561/0300000028

Frese, M., Erbe-Heinbokel, M., Grefe, J., Rybowiak, V., \& Weike, A. (1994). «I prefer to be told exactly what to do»- Acceptance of responsibility and job discretion in East and West Germany. Zeitschrift für Arbeits- und Organisationspsychologie, 38, 22-33.

Frese, M., \& Fay, D. (2001). Personal initiative (PI): An active performance concept for work in the 21 st century. En B. M. Staw \& R. M. Sutton (Eds.), Research in Organizational Behavior (Vol. 23, pp. 133-187). Amsterdam: Elsevier Science. doi:http://dx.doi.org/10.1016/S01913085(01)23005-6

Frese, M., Fay, D., Hilburger, T., Leng, K., \& Tag, A. (1997). The concept of personal initiative: Operationalization, reliability and validity in two German samples. Journal of Occupational and Organizational Psychology, 70, 139-161. doi:http://dx.doi.org/10.1111/j.20448325.1997.tb00639.x

Frese, M., Kring, W., Soose, A., \& Zempel (1996). Personal initiative at work: Differences between East and West Germany. Academy of Management Journal, 39, 37-63. doi: http://dx.doi.org/10.2307/256630
Frese, M., Teng, E., \& Wijnen, C. J. (1999). Helping to improve suggestion systems: Predictors of making suggestions in companies. Journal of Organizational Behavior, 20, 1139-1155. doi:http://dx.doi.org/10.1002/(SICI)10991379(199912)20:7<1139::AIDJOB946>3.0.CO;2-I

Frese, M., van Gelderen, M., \& Ombach, M. (2000). How to plan as a small-scale business owner: Psychological process characteristics of action strategies and success. Journal of Small Business Management, 38, 1-18.

Frese, M., \& Zapf, D. (1994). Action as the core of work psychology: A German Approach. En H. C. Triandis, M. D. Dunnette, \& L. M. Hough (Eds.), Handbook of industrial and organizational psychology (Vol. 4, pp. 271340). Palo Alto, CA: Consulting Psychology Press.

González, M. C., \& Tourón, J. (1992). Autoconcepto y rendimiento escolar. Sus implicaciones en la motivación y en la autorregulación del aprendizaje. Pamplona: EUNSA.

Hu, L. T., \& Bentler, P. M. (1999). Cutoff criteria for fit indexes in covariance structure analysis: Conventional criteria versus new alternatives. Structural Equation Modeling, 6, 1-55.

doi: http://dx.doi.org/10.1080/10705519909540118

Koop, S., De Reu, T., \& Frese, M. (2000). Sociodemographic factors, entrepreneurial orientation, personal initiative and environmental problems in Uganda. En M. Frese (Eds.), Success and Failure of Microbusiness Owners in Africa: A Psychological Approach (pp. 55-76). Westport, CT: Quorum.

Korunka, C., Frank, H., Lueger, M., \& Mugler, J. (2003). The entrepreneurial personality in the context of resources, environment, and the start-up process: A configurational approach. Entrepreneurship Theory and Practice, 28, 23-42.

doi:http://dx.doi.org/10.1111/15408520.00030

Krauss, S. I., Frese, M., Friedrich, C., \& Unger, J. M. (2005). Entrepreneurial orientation: A psychological model of success among 
Southern African small business owners. European Journal of Work and Organizational Psychology, 14, 315-344. doi: http://dx.doi.org/10.1080/1359432050017022 7

Lantz, A., \& Anderson, K. (2009). Personal initiative at work and when facing unemployment. Journal of Workplace Learning, 21, 88-108. doi: http://dx.doi.org/10.1108/1366562091093480 7

Lisbona, A., \& Frese, M. (2012). Iniciativa personal. Cómo hacer que las cosas sucedan. Madrid: Ediciones Pirámide.

Lisbona, A., Palací, F., \& Agulló, E. (2008). Escala de aspiraciones de control y responsabilidad: Adaptación española y su relación con la iniciativa personal. Psicothema, 20, 249-253.

Lisbona, A., Palací, F., \& Gómez, A. (2008). Escala de clima para la iniciativa y para la seguridad psicológica: Adaptación al castellano y su relación con el desempeño organizacional. Revista de Psicología del Trabajo y de las Organizaciones, 24 (2), 153167. doi: http://dx.doi.org/10.4321/s157659622008000200002

Lloret-Segura, S., Ferreres-Traver, A., HernándezBaeza, A., \& Tomás-Marco, I. (2014). El análisis factorial exploratorio de los ítems: una guía práctica, revisada y actualizada. Anales de Psicología, 30, 1151-1169. doi: http://dx.doi.org/10.6018/analesps.30.3.19936 1

Lumpkin, G. T., \& Dess, G. G. (1996). Clarifying the entrepreneurial orientation construct and linking it to performance. Academy of Management Review, 21, 135-172. doi: http://dx.doi.org/10.2307/258632

Marsh, H. W., Liem, G. A. D., Martin, A. J., Morin, A. J. S., \& Nagengast, B. (2011). Measurement fruitfulness of exploratory structural equation modelling (ESEM) in addressing key substantive issues in motivation and engagement research. Journal of Psychoeducational Assessment, 29, 322-346. doi: http://dx.doi.org/10.1080/09243453.2013.812568

Marsh, H. W., Morin, A. J. S., Parker, P. D., \& Kaur, G. (2014). Exploratory structural equation modeling: An integration of the best features of exploratory and confirmatory factor analysis. Annual Review of Clinical Psychology. doi: http://dx.doi.org/10.1146/annurev-clinpsy032813-153700

Martorell, C., González, R., Ordóñez, A., \& Gómez, O. (2011). Estudio confirmatorio del Cuestionario de conducta prosocial (Ccp) y su relación con variables de personalidad y socialización. Revista Iberoamericana de Diagnóstico y Evaluación - e Avaliação Psicológica, 32, 35-52.

Motowidlo, S. J., \& Scotter, J. R. V. (1994). Evidence that task performance should be distinguished from contextual performance. Journal of Applied Psychology, 79, 475-480. doi:http://dx.doi.org/10.1037//0021-9010.79.4.475

Peralta, S., Ramírez, A., \& Castaño, H. (2006). Factores resilientes asociados al rendimiento académico en estudiantes universitarios pertenecientes a la Universidad de Sucre. Psicología desde el Caribe, 196-219.

Ponton, M., \& Carr, P. (2002). The development of instrumentation that measures an adult's intention to exhibit initiative and resourcefulness in autonomous learning. En H. B. Long y Asociados (Eds.), Twenty-first century advances in self-directed learning (pp. 231-241). Schaumburg, IL: Motorola University Press.

Rank, J., Boedeker, N., Linke, M., \& Frese, M. (2004, Agosto). Integrating proactivity concepts into innovation research: The importance of voice and initiative. En S. K. Parker \& C. Collins (Coordinadores). Proactivity: Enhancing understanding of selfstarted and dynamic action within organizations. Simposio celebrado en el encuentro de la Academia de Gestión, Nueva Orleans.

Rank, J., Pace, V. L., \& Frese, M. (2004). Three avenues for future research on creativity, innovation, and initiative. Applied Psychology: An International Review, 53, 518-528.

doi:http://dx.doi.org/10.1111/j.14640597.2004.00185.x

Roth, E., \& Lacoa, D. (2009). Análisis psicológico del emprendimiento en estudiantes universitarios: Medición, 
relaciones y predicción. Revista Electrónica de Psicología, 7, 1-38.

Sanjuán, P., Pérez, A., \& Bermúdez, J. (2000). Escala de autoeficacia general: Datos psicométricos de la adaptación para la población española. Psicothema, 12, 509-513.

Velicer, W. F. (1976). Determining the number of components from the matrix of partial correlations. Psychometrika, 41, 321-327. doi: http://dx.doi.org/10.1007/BF02293557

Wolf, E. J., Harrington, K. M., Clark, S. L., \& Miller, M. W. (2013). Sample size requirements for Structural Equation Models: An evaluation of power, bias, and solution propriety. Educational and Psychological Measurement, 76, 913-934. doi: http://dx.doi.org/10.1177/0013164413495237

Zimmerman, B. J., \& Labuhn, A. (2012). Selfregulation of learning: Process approaches to personal development. En K.R. Harris, S. Graham, T. Urdan, C.B. McCormick, G.M. Sinatra, \& J. Sweller (eds.), APA educational psychology handbook: Theories, constructs, and critical issues (pp. 399-425). Washington, DC US: American Psychological Association. 